Steele, K. M., Dello Stritto, M. E., \& Brigner, W. L. (1997). Changes in perceived color with intermittent illumination. Perceptual and Motor Skills, 85(2): 759-765 (Oct 1997). Published by Ammons Scientific (ISSN: 0031-5125).

\title{
Changes in Perceived Color with Intermittent Illumination
}

Kenneth M. Steele, Mary Ellen Dello Stritto, Willard L. Brigner

\begin{abstract}
Using 24 observers with normal color vision, perceived shifts in hue were determined for a yellow-red, green, and blue-green at intermittencies of 5, 10, and $20 \mathrm{cps}$. The hue shift for yellow-red was consistent with the hue shift exhibited by a deuteranomalous observer while the hue shift for green and blue-green was consistent with that exhibited by a protanomalous observer.
\end{abstract}


Nelson, Bartley, and Mackavey (10) reported that persons with normal color vision exhibit color deficiencies when responding to certain Ishihara pseudoisochromatic plates under intermittent illumination. In additional reports $(4,9)$, anecdotal accounts have been presented of the changes in hue and saturation of several colors under various conditions of intermittent illumination. For example, changes in red have been described by one observer as becoming yellowish-red with an increase in intermittency and then becoming reddish amber as flicker fusion was approached (4, Table 3). If one assumes that brightness enhancement at $10 \mathrm{cps}$ (2) could effect a Bezold-Bruecke hue shift in the same way that the hue shift can be effected by an increase in light intensity, then a shift towards yellow, a yellowish-red, would be predicted at an intermittency of around $10 \mathrm{cps}$. Then, as flicker fusion is approached, a drop in intensity develops in accordance with Talbot's law (vid. 3), and there would be a shift in perceived hue towards red (1) which would perhaps correspond to the observer's report of reddish amber. In other words, changes in perceived hue under intermittent illumination may possibly be accounted for in terms of the Bezold-Bruecke shift in perceived hue which occurs with changes in light intensity, viz., shifts toward yellow and blue as apparent brightness increases and shifts toward red and green as apparent brightness decreases.

Furthermore, Nelson, et al.'s (10) report of color deficiency occurring under intermittent illumination appears related to these proposed hue shifts, since there are similarities between the Bezold-Bruecke hue shift and the changes in perceived hue occurring in the most frequently reported color deficiencies, protanomaly and deuteranomaly. Consider, for example, the hue shifts for orange. With an increase in light intensity, orange shifts towards yellow and appears more yellowish to the observer with normal color vision. Similarly, the deuteranomalous observer requires more yellow to match orange than the normal observer (8). As light intensity decreases, orange shifts towards red and appears more reddish; similarly, the protanomalous observer requires more red in matching orange than the normal observer (8). As a further example, consider the hue shifts of blue-green or cyan. As intensity increases, the hue increases in blueness for the normal observer; the deuteranomalous observer also requires more blue in matching cyan than the normal observer. As intensity decreases, cyan is perceived as more greenish by the normal observer. Similarly, the protanomalous observer requires more green in matching cyan than the normal observer (8). Clearly, hue shifts with changes in light intensity can mimic the hue shifts seen in the color deficiencies protanomaly and deuteranomaly. Furthermore, it seems likely that brightness enhancement at an intermittency of around $10 \mathrm{cps}$ could effect the hue shifts which ordinarily occur with increased light intensity. Although the hue shifts in protanomaly and deuteranomaly occur across the entire visible spectrum, there are three invariant points in the spectrum when hue shifts are effected by intensity changes. These invariant points are at about 478, 503, and $572 \mathrm{~nm}$ (12). Therefore, if a hue shift is to be considered a Bezold-Bruecke hue shift, a green corresponding to $503 \mathrm{~nm}$, for example, should not be perceived as changed in hue at an intermittency that is associated with brightness enhancement or with increased apparent brightness. 
The purpose of the current experiment was twofold: (1) to determine quantitative changes in hue or saturation occurring across three illumination intermittencies for three colors and thereby (2) to test the prediction that the Bezold-Bruecke hue shift is associated with brightness enhancement-a hue shift which can mimic the color deficiencies of protanomaly and deuteranomaly and thus account for the deficiency in color vision which has been reported for intermittent illumination.

\section{METHOD}

\section{Observers}

Six graduate students and 18 undergraduates were observers (13 women and 11 men). Their participation earned course credit. All had normal color vision as evaluated by Dvorine pseudoisochromatic plates.

\section{Stimuli}

Stimuli were matte Munsell papers corresponding to an orange with an OSA dominant wavelength 2 of $590 \mathrm{~nm}$ (5YR 518), a green having an OSA dominant wavelength of $509 \mathrm{~nm}$ (5G 5/8), and a cyan having an OSA dominant wavelength of $489 \mathrm{~nm}$ (7.5BG 5/8). These were viewed in a 2-channel tachistoscope (Scientific Prototype Model 800F). Viewing conditions were continuous illumination and three different rates of intermittency, viz., $5 \mathrm{cps}$, $10 \mathrm{cps}$, and $20 \mathrm{cps}$ with a pulse to cycle fraction (PCF) of .25. Note that the range of intermittencies brackets the intermittency of $10 \mathrm{cps}$ at which brightness enhancement occurs. The PCF of .25 corresponds to the PCF at which Nelson and Bartley (9) reported maximum changes in hue and saturation. The luminance of both the intermittent fields and continuous field was 2.3 foot Lamberts as measured at the eyepiece by spotmeter. The ambient luminance of a Munsell book of color was 4.5 foot Lamberts as measured by spotmeter at the observer's viewing distance.

\section{Procedure}

There were four illumination conditions-continuous illumination and intermittent illuminations at 5,10 , and $20 \mathrm{cps}$, and there were three different Munsell color conditions-5YR 5/8, 5G 5/8, and 7.5BG 5/8. These 12 stimulus combinations were counterbalanced across observers in accordance with a balanced square design.

Observers matched each of the three Munsell colored papers under each of the four illumination conditions by choosing a match from the Munsell book of color which was placed on a table next to the tachistoscope. J The organization of the Munsell book of color was demonstrated to the observer, and the observer was encouraged to look through the book to become more familiar with its organization. Observers were instructed to find the best possible match. They were instructed to pay particular attention to slight color changes, to take as much time as needed, and to feel free to flip through the pages of the Munsell book until a accurate match was found. Once a match was chosen, the hue, value, and chroma of the match was recorded. 


\section{RESULTS}

The Munsell papers chosen by observers to match the colors presented in the tachistoscope were converted from Munsell notation to $\mathrm{ICl} x$ and $y$ chromaticity coordinates by reference to Newhall, Nickerson, and Judd's Table 1 (11). The $x$ and $y$ chromaticity coordinates were then used to determine the dominant wavelength of the matching color by using the procedure outlined by Geldard (7). Averaging across the dominant wavelengths of the color matches of 24 observers, the average dominant wavelength of each matching color was determined for each of the four illumination conditions and for each of the three Munsell colors. The averages are presented in Fig.

1.

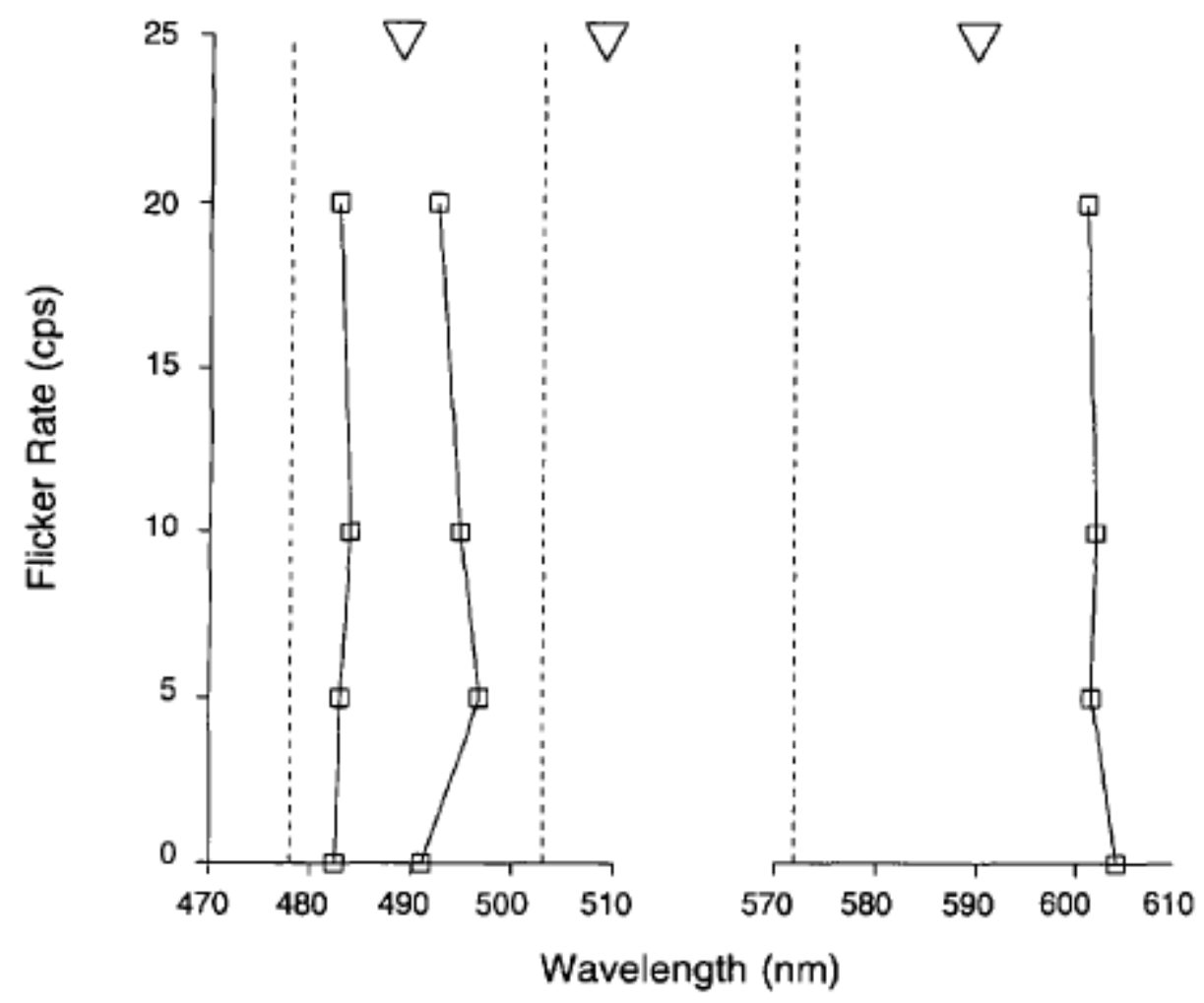

FIG. 1. Changes in matched color across different flicker rates. The ordinate represents flicker rate conditions with continuous illumination indicated as zero flicker rate. The abscissa shows stimuli and judgments arranged with respect to wavelength. Vertical dashed lines indicate wavelength points invariant to hue shifts induced by intensity changes. Downward points along the top indicate location of the OSA dominant wavelength\} of the three Munsell papers used as stimuli. The break in the abscissa was used to reduce the width of the figure.

First, consider the color matches to 7.5BG 5/8 which has an OSA dominant wavelength2 of 489 run. When viewed in the tachistoscope under continuous illumination, the latter was matched by papers from the Munsell book of color which had an average dominant wavelength of $482.47 \mathrm{~nm}$. This discrepancy between OSA dominant wavelength and the matched dominant 
wavelength was no doubt due to differences in viewing conditions and illuminants, and it was, of course, not of major importance to the current investigation. Of major interest was the change in color matches, if any, when illumination was intermittent versus continuous. As shown in Fig. 1, at an intermittency of $5 \mathrm{cps}$, the average dominant wavelength of the color matches to $7.5 \mathrm{BG} 5 / 8$ was $482.98 \mathrm{~nm}$-a slight shift towards green as compared to the average dominant wavelength of $482.47 \mathrm{~nm}$ with continuous illuminetion. At an intermittency of $10 \mathrm{cps}$, the average dominant wavelength of the color matches was $484 \mathrm{~nm}$-a somewhat greater shift towards green than with continuous illumination or with an intermittency of $5 \mathrm{cps}$. Finally, at an intermittency of $20 \mathrm{cps}$, the average dominant wavelength was $482.8 \mathrm{~nm}-\mathrm{a}$ slight shift towards green as compared to the average dominant wavelength when illumination was continuous. Note that intermittency led to an average dominant wavelength of color matches which was shifted towards green as compared to the color matches under continuous illumination.

Next, consider the color matches to 5G 5/8 which has an OSA dominant wavelength2 of $509 \mathrm{~nm}$. As viewed in the continuous illumination of the tachistoscope, 5G 5/8 was matched by Munsell papers having an average dominant wavelength of $491.1 \mathrm{~nm}$. The discrepancy between OSA dominant wavelength and matched dominant wavelength was, as noted above, no doubt due to differences in illuminants and viewing conditions. As shown in Fig. 1, at an intermittency of $5 \mathrm{cps}$, the average dominant wavelength of the color matches was $496.8 \mathrm{~nm}$-a shift towards green as compared to the average dominant wavelength of $491.1 \mathrm{~nm}$ under continuous illumination. At an intermittency of $10 \mathrm{cps}$, the average dominant wavelength of the color matches was $494.9 \mathrm{~nm}$, again a shift towards green with intermittency but a smaller shift than occurred at $5 \mathrm{cps}$. Finally, at an intermittency of $20 \mathrm{cps}$, the average dominant wavelength of the color matches was $492.6 \mathrm{~nm}$-another shift towards green but a smaller shift than occurred at intermittencies of 5 or $10 \mathrm{cps}$. As with color matches to 7.5BG 5/8, the average dominant wavelength of color matches to $5 G$ 5/8 under intermittent illumination was shifted towards green as compared to the color matches under continuous illumination.

Finally, consider the color matches to 5YR 5/8 which has an OSA dominant Wavelength [2] of $590 \mathrm{~nm}$. When viewed in the tachistoscope under continuous illumination. 5YR 5/8 was matched by Munsell papers having an average dominant wavelength of $604 \mathrm{~nm}$. As noted above, differences in illuminants and in viewing conditions were probably the basis for the discrepancy. As presented in Fig. 1, at an intermittency of $5 \mathrm{cps}$, the average dominant wavelength of the color matches to 5 YR $5 / 8$ was $601.5 \mathrm{~nm}$-a shift towards yellow as compared to the average dominant wavelength of $604 \mathrm{~nm}$ under continuous illumination. At an intermittency of $10 \mathrm{cps}$, the average dominant wavelength of the color matches was $602 \mathrm{~nm}$-a somewhat smaller shift towards yellow than with an intermittency of $5 \mathrm{cps}$. Lastly, at an intermittency of $20 \mathrm{cps}$, the average dominant wavelength of the color matches was $601 \mathrm{~nm}$-another shift towards yellow and a somewhat greater shift than occurred at the intermittencies of 5 and $10 \mathrm{cps}$. 
Clearly, these data are inconsistent with the initial hypothesis of a systematic Bezold-Bruecke hue shift towards blue and yellow as a result of brightness enhancement at an intermittency of $10 \mathrm{cps}$. [4] Although there was a shift towards yellow with 5YR 5/8, the maximum shift occurred at an intermittency of $20 \mathrm{cps}$, and there is no basis for anticipating a brightness increase at this intermittency which would enable one to predict a hue shift. On the other hand, there appears to be a relatively simple systematic account for the current data. Since additive mixing of green and red yields yellow, the data of the current investigation can be accounted for theoretically by assuming that intermittency results in the addition of green subjective/illusory color. The addition of illusory/subjective green would account for the shift towards yellow with 5YR 5/8 and the shifts towards green with 7.5BG $5 / 8$ and $5 G 5 / 8$. While illusory color is usually produced with black-white stimuli, illusory green has been reported with colored stimuli and at a wide range of intermittencies (vid. 5). The intermittency-induced shift towards yellow with 5 YR $5 / 8$ is, as presented earlier, characteristic of the deuteranomalous observer who requires more yellow in matching long wavelengths than the normal observer (8). The intermittency-induced shift towards green with 7.5BG $5 / 8$ and $5 G 5 / 8$ is characteristic of the protanomalous observer who requires more green to match short wavelengths than the normal observer (8). Although the hue shifts seen in deuteranomaly and protanomaly are not represented systematically by the current data, the current data suggest that intermittency could contribute illusory color which would result in a normal observer exhibiting behaviors consistent with color deficiency.

\section{NOTES}

2. "OSA dominant wavelength" refers to the dominant wavelength as determined from specifications provided by the Optical Society of America (OSA) subcommittee studying the Munsell colors (11) under illumination and viewing conditions not replicated here.

3. The procedure has been used previously by White, Lockhead. and Evans (13) and is an adaptation of the graded series method as presented by Coren and Girgus (6).

4. Although the reported hue shifts were not systematically consistent with the Bezold-Bruecke hue shift, the hue shifts were generally of the same magnitude as those expected with the Bezold-Bruecke phenomenon for the perceived hues investigated (12). Also, note that, for a given perceived hue, there was an orderly, systematic hue shift in one direction occurring with intermittency. As a consequence of this systematic shift In hue, the reported hue shifts provide a coherent account for me color deficiency reported by Nelson, et al. (10) as clarified in the text. 


\section{REFERENCES}

1. ABNEY, W. DE W. Researches in color vision. London: Longmans, Green, 1913.

2. BARTLEY, S. H. Some factors in brightness discrimination. Psychological Review, 1939, 46, 337-358.

3. BARTLEY, S. H. The psychophysiology of vision. In S. Stevens (Ed.), Handbook of experimental psychology. New York: Wiley, 1963. Pp. 921-984.

4. BARTLEY, S. H., \&NELSON, T. M. Certain chromatic and brightness changes associated with rate of intermittency of photic stimulation. Journal of Psychology, 1960, 50, 323-332.

5. COHEN, J .. \&GORDON, D. A. The Prevost-Fechner-Benham subjective colors. Psychological Bulletin, 1949, 46,97-136.

6. COREN, S., \& GIRGUS, J. S. A comparison of five methods of illusion measurement. Behavior Research Methods and Instrumentation, 1972,4, 240-244.

7. GELDARD, E A. The human senses. New York: Wiley, 1962. Pp. 61-63.

8. HURVICH, L. M., \&JAMESON, D. An opponent-process theory of color vision. Psychological Review, 1957, 64, 384-404.

9. NELSON, T. M., \&BARTLEY, S. H. The role of PCF in temporal manipulation of color. Journal of Psychology, 1961,52,457-477.

10. NELSON, T. M., BARTLEY, S. H., \& MACKAVEY, W. R. Responses to certain pseudoisochromatic charts viewed in intermittent illumination. Perceptual and Motor Skills, $1961,13,227-231$.

11. NEWHALL, S. M., NICKERSON, D., \& JUDD, D. B. Final report of the O.S.A. subcommittee on the spacing of the Munsell colors. Journal of the Optical Society of America, 1943, 33, 385-418.

12. PURDY, D. M. The Bezold-Bruecke phenomenon and contours for constant hue. American Journal of Psychology, 1937,49, 313-315.

13. WHITE, C. W, LOCKHEAD, G. R., \& EVANS, N. J. Multidimensional scaling of subjective colors by color-blind observers. Perception and Psychophysics, 1977, 21, 522-526. 